TECHNICAL NOTE

\author{
E. Elolf \\ V. Bockermann \\ T. Gringel \\ M. Knauth \\ P. Dechent \\ G. Helms
}

\title{
Improved Visibility of the Subthalamic Nucleus on High-Resolution Stereotactic MR Imaging by Added Susceptibility (T2*) Contrast Using Multiple Gradient Echoes
}

SUMMARY: Reliable identification of the subthalamic nucleus (STN) is a critical step in deep brain stimulation for Parkinson disease but difficult on T1-weighted stereotactic MR imaging. By simultaneous imaging of multiple gradient echoes, susceptibility contrast is added to conventional T1weighted high-resolution MR image. Thus, the visibility of the STN is enhanced on a second colocalized dataset by exploiting the sensitivity of the $T 2^{*}$-relaxation to local iron deposits. The feasibility is underpinned by quantitative measurements on healthy adults.

A $\mathrm{n}$ established neurosurgical therapy for Parkinson disease is to place electrodes for deep brain stimulation within the subthalamic nucleus (STN). ${ }^{1}$ Reliable identification of the anatomic borders of the STN is thus a critical step in stereotactic procedures. $^{2,3}$ T2-weighted MR imaging showing the iron-rich structures as hypointensities has been suggested as additional information to the poor contrast of the STN on T1-weighted highresolution 3D MR imaging. ${ }^{4,5}$ Exploiting the increased sensitivity of $\mathrm{T}^{*}$ to local iron deposits, a multigradient echo fast low-angle shot (FLASH) technique ${ }^{6}$ is proposed to visualize the STN. This 3D MR technique enables simultaneous acquisition of T1weighted images for stereotactic use and images with superimposed $\mathrm{T} 2{ }^{\star}$ contrast to localize the STN.

\section{Technique}

The feasibility study was carried out on 16 healthy adults ( 8 men; age range, 23-31 years; mean age, 26 years) on a 3T MR system (Magnetom Trio; Siemens Medical Solutions, Erlangen, Germany) using an 8-channel phased-array head coil (MR Imaging Devices, Waukesha, Wis). Written informed consent as supervised by the local ethical committee was obtained.

A multi-echo FLASH sequence (TR, $30 \mathrm{~ms}$; flip angle, $20^{\circ}$ ) provided primarily $\mathrm{T} 1$-weighted $3 \mathrm{D}$ datasets of $0.95-\mathrm{mm}$ isotropic resolution $(\mathrm{FOV}=243 \mathrm{~mm}$; 176 sagittal partitions with 6/8 partial Fourier sampling in phase and section directions; 7:09 minutes). Eight gradient-echoes $(\mathrm{TE}=2.2 / 5.2 / 8.2 / 11.2 / 14.2 / 17.2 / 20.2 / 23.2 \mathrm{~ms}$; bandwidth/pixel $=370 \mathrm{~Hz}$ ) provided additional $\mathrm{T}^{*}$-weighted contrast increasing with TE. For comparison, multisection turbo spinecho images (TSE; 29 contiguous 2-mm axial sections; effective TE, $119 \mathrm{~ms}$; TR, $3900 \mathrm{~ms}$ ) were also obtained.

The data were transferred to a stereotactic workstation (Sofamore Danek Stealth Station; Medtronic, Minneapolis, Minn) for coregistration of images with the Schaltenbrand-Wahren atlas. ${ }^{7}$ For quantitative comparison, $\mathrm{T}^{*}$ was determined in the STN and the reticulate formation by a region-of-interest analysis in 1 subject.

Received December 15, 2006; accepted after revision January 31, 2007

From the Department of Neuroradiology (E.E., T.G., M.K.), Department of Neurosurgery (V.B.), and MR-Research in Neurology and Psychiatry (T.G., P.D., G.H.), Georg-AugustUniversität Göttingen, Göttingen, Germany.

This work was supported by the Volkswagen Foundation of Lower Saxony.

Address correspondence to Gunther Helms, MR-Forschung in der Neurologie und Psychiatrie, Universitätsklinikum, D-37099 Göttingen, Germany; e-mail: ghelms@gwdg.de DOI 10.3174/ajnr.A0527

\section{Results}

The $\mathrm{T} 2^{*}$-signal intensity decay was markedly faster in the area of the STN than in the surrounding tissue. Figure 1 shows representative curve fits to the STN and the reference region of the reticulate formation. This corresponded with a contrast of approximately $25 \%$ at the longest TE. Thus, both STNs could be identified in all 16 of the subjects even when motion artifacts impaired the delineation (2 subjects).

Figure 2 shows the midbrain region, where the solely T1weighted data (echo 1 ; TE $=2.3 \mathrm{~ms}$; top) did not reveal any structures, but the STN became clearly visible at a TE of $20 \mathrm{~ms}$ and above (echo 7; middle). The main axis of the STN was slightly oblique to the anterior/posterior commissure plane shown in Fig 2. Its position was approximately $7 \mathrm{~mm}$ rostroanterior to the superior pole of the red nucleus. The spatial assignment was confirmed by comparison with the coregistered T2-weighted TSE images with the use of a stereotactic system. In the subject shown in Fig 2, the estimated position of the STN by using Schaltenbrand-Wahren coordinates was slightly different from the direct visually identified position. In addition, phase data were acquired to calculate the spatial distortions as a result of magnetic field inhomogeneities. Even in the critical areas at the skull base, these did not exceed 1 pixel.

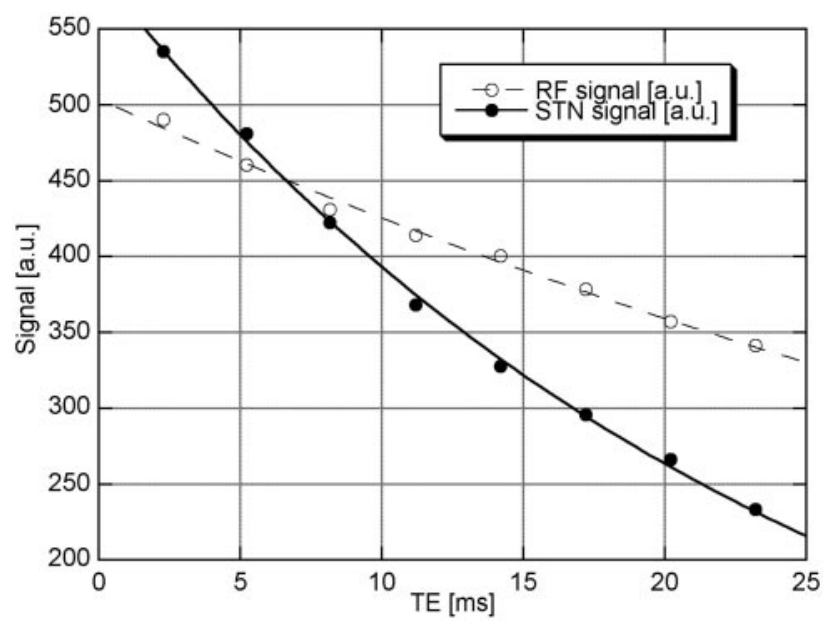

Fig 1. T2* decay in the STN (dots) and reticular formation (RF; circles). Fitted T2* values were $25.0 \pm 0.4 \mathrm{~ms}$ (STN, bold) and $59.1 \pm 2.0$ (RF, dashed). 


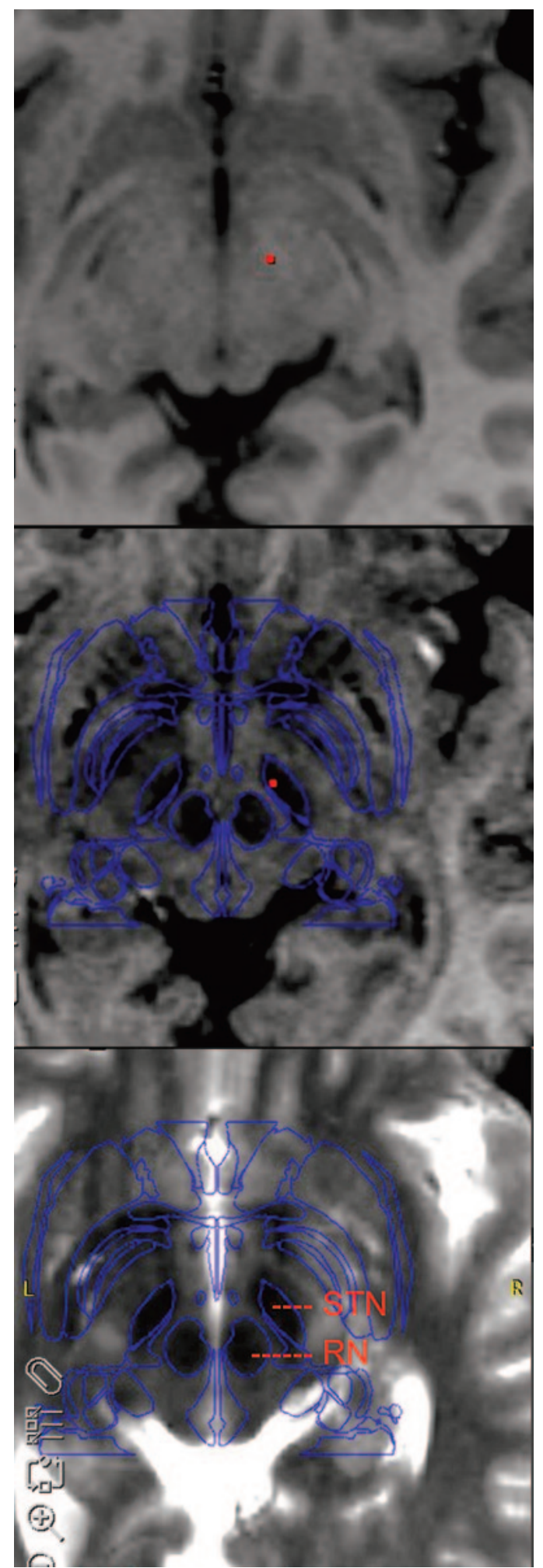

Fig 2. Comparison of $\mathrm{T} 1$-weighted, $\mathrm{T} 1+\mathrm{T} 2^{*}$-weighted, and $\mathrm{T} 2$-weighted (top to bottom) MR imaging in anterior/posterior commissure orientation. Note the $\mathrm{T} 2^{*}$ contrast of vessels and iron-containing structures. The outlines of the Schaltenbrand-Wahren atlas are shown as overlay (blue). The STN and red nucleus (RM) are assigned. A mismatch between the atlas and individual anatomy of the STN can be seen. The apparent inconsistency between T2*and T2-weighted images (as seen on the left and right STN) demonstrates the limitations when coregistering the multisection T2-weighted data with the multiecho 3D datasets.

\section{Discussion}

The correlation of iron deposits in the STN and the associated hypointensities on T2-weighted MR imaging has been firmly established by a histologic study ${ }^{4}$ and has been applied recently at $3 \mathrm{~T}^{5}$ This is the first report to use the higher sensitivity of $\mathrm{T}^{*}$ to local iron content ${ }^{8}$ for direct visualization of the STN. Comprehensible contrast could be obtained at short TE compatible with TR durations as used in high-resolution 3D T1-weighted MR imaging in clinically feasible time. We were able to demonstrate the $\mathrm{T} 2^{*}$ contrast of the STN also at $1.5 \mathrm{~T}$ by using a standard quadrature head coil and a single echo measurement with TE at $20 \mathrm{~ms}$. Implementation as a multiecho technique permitted simultaneous acquisition and, thus, inherently co-localized datasets without loss of resolution such as T2-weighted MR imaging and without additional measuring time. The dataset of the first echo may be used in standard stereotactic procedures; the later echoes provide additional contrast of iron-containing structures. The low heat deposition of the gradient-echo approach is an additional advantage at higher field strengths and in the presence of metal implants. However, the feasibility for postsurgical control is expected to be hampered by the presence of $B_{0}$ field inhomogeneity. Quantitative in vivo data of the iron content and relaxation in the STN are lacking, because this structure has not been evaluated in MR imaging studies. ${ }^{8,9}$ Further systematic evaluation on a larger cohort is in progress to fully evaluate the potential of the suggested technique and individual-, age-, and diseaserelated variations.

\section{Conclusion}

Because of the iron content of the STN and sensitivity of $2^{\star}$ to iron deposits, the STN can be visualized on stereotactic MR imaging by simultaneous acquisition of multiple gradient echoes at $3 \mathrm{~T}$ to generate co-localized $3 \mathrm{D}$ datasets with $\mathrm{T} 1$ and $\mathrm{T} 1-\mathrm{T} 2{ }^{\star}$ contrast.

\section{Acknowledgments}

We thank Dr G. Krüger of Siemens Medical Solutions for providing the multiecho FLASH sequence.

\section{References}

1. Richter EO, Hoque T, Halliday W, et al. Determining the position and size of the subthalamic nucleus based on magnetic resonance imaging results in patients with advanced Parkinson disease. J Neurosurg 2004;100:541-46

2. Aziz TZ, Nandi D, Parkin S, et al. Targeting the subthalamic nucleus. Stereotact Funct Neurosurg 2001;77:87-90

3. Benabid AL, Koudsie A, Benazzouz A, et al. Subthalamic stimulation for Parkinson's disease. Arch Med Res 2000;31:282-89

4. Dormont D, Ricciardi KG, Tande D, et al. Is the subthalamic nucleus hypointense on T2-weighted images? A correlation study using MR imaging and stereotactic atlas data. AJNR Am J Neuroradiol 2004;25:1516-23

5. Slavin KV, Thulborn KR, Wess C, et al. Direct visualization of the human subthalamic nucleus with $3 \mathrm{~T}$ MR imaging. AJNR Am J Neuroradiol 2006;27:80-84

6. Frahm J, Haase A, Matthaei D. Rapid three-dimensional MR imaging using the FLASH technique. J Comput Assist Tomogr 1986;10:363-68

7. Schaltenbrand G, Wahren W. Atlas for Stereotaxy of the Human Brain. Stuttgart, Germany: Thieme; 1977

8. Gelman N, Gorell JM, Barker PB, et al. MR imaging of human brain at 3.0 T preliminary report on transverse relaxation rates and relation to estimated iron content. Radiology 1999;210:759-67

9. Schenck J, Zimmerman E. High field magnetic resonance imaging of brain iron: birth of a biomarker? NMR Biomed 2004;17:433-45 